\title{
STATIONARY ISOTOPIES OF INFINITE-DIMENSIONAL SPACES
}

\author{
BY \\ RAYMOND Y. T. WONG
}

\begin{abstract}
Let $X$ denote the Hilbert cube or any separable infinite-dimensional Fréchet space. It has been shown that any two homeomorphisms $f, g$ of $X$ onto itself is isotopic to each other by means of an invertible-isotopy on $X$. In this paper we generalize the above results to the extent that if $f, g$ are $K$-coincident on $X$ (that is, $f(x)=g(x)$ for $x \in K)$, then the isotopy can be chosen to be $K$-stationary provided $K$ is compact and has property-Z in $X$. The main tool of this paper is the Stable Homeomorphism Extension Theorem which generalizes results of Klee and Anderson.
\end{abstract}

1. An invertible-isotopy of space $X$ to space $Y$ is a homeomorphism $F$ of $X \times I$ onto $Y \times I$ such that $F(X \times t)=Y \times t$ for all $t \in I=[0,1]$. We denote such an $F$ by $\left\{f_{t}=\left.F\right|_{X \times t}\right\}_{t \in I}$. For $K \subset X, F$ is $K$-stationary if for each $x \in K, f_{t}(x)=f_{0}(x)$ for all $t$. The main results of this paper are Theorem 1.1 and Theorem 2.1.

THEOREM 1.1. If $X$ is the Hilbert cube or $s$ (on any separable infinite-dimensional Fréchet space) and $K$ is a compact set with property- $\mathrm{Z}$ in $X$, then any two $K$-coincident homeomorphisms $f, g$ of $X$ onto $X$ are isotopic by an invertible-isotopy $F$ such that $F$ is of $K$-stationary.

Two maps $f_{1}, f_{2}: X \rightarrow Y$ are $A$-coincident, $A \subset X$, if $f_{1}(x)=f_{2}(x)$ for all $x \in A$. A closed set $A$ of $X$ has property-Z in $X$ (following Anderson [2]) if for each homotopically trivial nonnull open subset $U$ of $X, U \backslash A$ is both nonnull and homotopically trivial.

The Hilbert cube $Q$ is the infinite product space $\prod_{i=1}^{\infty} J_{i}$ and $s$ is the space $\prod_{i=1}^{\infty}$ Int $J_{i}$, where $J_{i}=J=[-1,1] . s$ is homeomorphic to any separable infinitedimensional Fréchet space [0]. We consider $s$ as imbedded canonically in $Q, s$ is sometimes called the pseudointerior (PI) of $Q$. Let $B(Q)=Q \mid s$. A homeomorphism of $Q$ onto itself is a $\beta^{*}$-homeomorphism (following Anderson [2]) if $h(s)=s$. Let $G(X)$ denote the group of all homeomorphisms of $X$ onto itself and let $G^{*}(Q)$ denote all $\beta^{*}$-homeomorphisms of $G(Q)$. A set $K$ in $Q$ is Core if $K$ has the form $\prod_{i=1}^{\infty}\left[-a_{i}, a_{i}\right]$ where each $0<a_{i}<1$ and $\left[-a_{i}, a_{i}\right] \subset \operatorname{Int} J_{i}$. Theorem 1.1 follows from the following.

Presented to the Society, January 23, 1970; received by the editors October 14, 1969.

AMS 1969 subject classifications. Primary 5460, 5755; Secondary 5485.

Key words and phrases. Invertible-isotopy, $K$-coincident, $K$-stationary, homeomorphism, property-Z, stable homeomorphism extension. 
THeOREM 1.2. Let $X=Q$ or $s$ and let $K$ be a compact set with property-Z in $X$. If $h$ is a homeomorphism of $X$ onto $X$ such that $\left.h\right|_{K}=$ identity, then there is an invertibleisotopy $\left\{h_{t}\right\}$ of $X$ onto itself such that $h_{1}=h, h_{0}=$ identity and $\left.h_{t}\right|_{K}=$ identity for all $t$.

Proof of Theorem 1.1. Let $f, g$ be given as in Theorem 1.1. Consider $h=f^{-1} g$. Then $\left.h\right|_{K}=$ identity. Let $\left\{h_{t}\right\}$ be given by Theorem 1.2. Then $\left\{f \cdot h_{t}\right\}$ is an invertibleisotopy between $f$ and $g$.

The author wants to thank the referees for their elaborate comments. In particular, we thank Mr. Barit for pointing out an alternative, but simpler argument for Theorem 2.1, whose proof we shall adopt here.

\section{Stable Homeomorphism Extension Theorem.}

THEOREM 2.1. Let $X=Q$ or $s$ and let $K$ be a compact set in $X \times I$ such that $K \cap X \times t \neq \varnothing$ for all $t$ and for $X=Q, K \subset s \times I \subset Q \times I$. Let $h$ be a homeomorphism of $K$ into $X \times I$ such that (1) $h(K \cap X \times t) \subset X \times t$ for all $t$, (2) $\left.h\right|_{K \cap X \times\{0,1\}}=$ identity and (3) for $X=Q, h(K) \subset s \times I$.

Then there is an $h_{1} \in G(X \times I)$ such that

(a) $h_{1}(X \times t)=X \times t$ for all $t$,

(b) $\left.h_{1}\right|_{K}=h$ and

(c) $\left.h_{1}\right|_{x \times\{0,1\}}=$ identity.

Following the notations of Theorem 2.1, we have

Corollary 2.2. If $X, K$ and $h$ are given as above satisfying (1), (3) and (2'): $\left.h\right|_{K \cap X \times 1}=\left.f\right|_{K \cap X \times 1}$ and $\left.h\right|_{K \cap X \times 0}=\left.f^{\prime}\right|_{K \cap X \times 0}$ where $f$ and $f^{\prime}$ are given homeomorphisms on $X \times 1$ and $X \times 0$ respectively, and when $X=Q$, $f$ and $f^{\prime}$ are $\beta^{*}$-homeomorphisms. Then there is an $h_{1} \in G(X \times I)$ such that $h_{1}$ satisfies (a), (b), and

(c') $\left.h_{1}\right|_{X \times 1}=f$ and $\left.h_{1}\right|_{X \times 0}=f^{\prime}$.

Proof. By [7] there is an invertible-isotopy $F=\left\{f_{t}\right\}$ of $X$ onto itself such that $f_{1}=f, f_{0}=f^{\prime}$ and for $X=Q$, each $f_{t}$ is $\beta^{*}$. Consider $h^{\prime}=F^{-1} h . h^{\prime}$ satisfies the hypothesis of Theorem 2.1 and let $h_{1}^{\prime}$ be given by the theorem for $h^{\prime}$. Then $h_{1}=F h_{1}^{\prime}$ is the desired function.

Corollary 2.3. If $X, K$ and $h$ are given as above satisfying (1), (3) of the hypothesis, then there is an $h_{1} \in G(X \times I)$ satisfying (a) and (b).

(This corollary can also be proved directly from the procedures used in [1].)

Proof. By [1, Theorem 4.2] there are homeomorphisms $f, f^{\prime}$ of $X$ onto itself such that $\left.f\right|_{K \cap X \times 1}=\left.h\right|_{K \cap(X \times 1)}$ and $\left.f^{\prime}\right|_{K \cap X \times 0}=\left.h\right|_{K \cap X \times 0}$ and for $X=Q, f$ and $f^{\prime}$ are $\beta^{*}$. homeomorphisms. Now apply Corollary 2.2.

QUESTION 1. Can the compact set $K$ be replaced by a closed set with property-Z in Theorem 1.1 ?

As it stands Theorem 2.1 cannot be generalized to an arbitrary closed $Z$-set in $s \times I$. A simple example would be to consider $K=C \times\{0\} \subset s \times J$ where $C$ is a wild 
0 -dimensional closed set in $s$ [8]. It is not difficult to see that $K$ is a closed $Z$-set in $s \times J$. But a level-preserving imbedding of $K$ into $s \times J$ that pushes $C$ inside $s \times J$ obviously has no extension. A more meaningful question would be: In Theorem 2.1 , can $K$ be replaced by a closed set which has property- $Z$ at each level? The answer to this question is yes. In a subsequent paper the author gives a solution to the question which appeared as a special case of a more general theorem.

Theorem 2.1 may be regarded as a stable generalization of homeomorphism extension theorems of V. Klee [5] for $X=l_{2}$ and of Anderson [1], [2]. Before we prove Theorem 1.2, we need the following.

3. LEMMA 3.1. Let $h$ be any homeomorphism of $Q$ onto $Q$ such that for some compact subset $K$ of $s, h(K)=K$. Then there is a sub-Hilbert cube $K^{\prime} \subset s$ such that $K \subset K^{\prime}$ and $h\left(K^{\prime}\right) \subset s$.

Proof. Let $\pi_{i}$ denote the projection mapping of $Q$ into the $i$ th coordinate. Write $Q \mid s$ as $K_{1} \cup K_{2} \cup \cdots$ where each $K_{i}=\pi_{j}^{-1}(-1)$ or $\pi_{j}^{-1}(1)$ for some $j$. Let $G$ denote the space of all imbeddings of $Q$ into $Q$ under the usual supremum metric. $G$ is completable [6, p. 32]. Let $G^{\prime}$ be the subset of $G$ consisting of all $f \in G$ such that $f(K)=K$. It is easy to see that $G^{\prime}$ is closed in $G$. Hence is of 2nd-category. Let $G_{i}=\left\{f^{\prime} \in G^{\prime} \mid f^{\prime}(Q) \cap\left(K_{i} \cup h\left(K_{i}\right)\right)=\varnothing\right\}$. We claim that each $G_{i}$ (namely $G_{1}$ ) is both open and dense in $G^{\prime} . G_{1}$ is clearly open. To show $G_{1}$ is dense, suppose $g \in G^{\prime}$ and $\varepsilon>0$. Consider $h^{-1} g(Q), h^{-1} g(K)=K$. Since $K \cap K_{1}=\varnothing$, by moving the endpoints of the first coordinate interval inside a little bit, it is clear that there is a $g_{1} \in G^{\prime}$ such that $g_{1}\left(h^{-1} g(Q)\right) \cap K_{1}=\varnothing$. Hence $h\left[g_{1} h^{-1} g(Q)\right] \cap h\left(K_{1}\right)=\varnothing$. Similarly there is a $g_{2} \in G^{\prime}$ such that $g_{2}\left[h g_{1} h^{-1} g(Q)\right] \cap\left(K_{1} \cup h\left(K_{1}\right)\right)=\varnothing$. Since $g_{1}, g_{2}$ can be chosen arbitrarily small, we can get $f^{\prime}=\left(g_{2}\right)\left(h g_{1} h^{-1}\right) g$ arbitrarily close to $g$ or $d\left(f^{\prime}, g\right)<\varepsilon$. This shows $G_{1}$ is dense and the proof of the claim is complete. Now let $f \in \bigcap_{i \geqq 1} G_{i}$. Hence $f(Q) \subset s$ and $f(Q) \cap h(Q \mid s)=\varnothing$. Let $K^{\prime}=h^{-1}(f(Q))$. $K^{\prime}$ has the desired properties.

Alternative proof. Note that $A=B\left(I^{\infty}\right) \cup h^{-1}\left(B\left(I^{\infty}\right)\right)$ misses $K . Q \backslash A \cong s \quad[3$, Theorem II]. Thus there is a sub-Hilbert cube $K^{\prime}$ of $Q \backslash A$ containing $K . K^{\prime}$ is also a sub-Hilbert cube of $Q, K^{\prime} \subset s$ and $h\left(K^{\prime}\right) \subset s$.

Proposition 3.2 (ANDERSON [2]). For $X=Q$ or $s$, any homeomorphism between two closed sets each with property- $\mathrm{Z}$ in $X$ can be extended to a homeomorphism of $X$ onto $X$. Furthermore, if $X=Q$ and both closed sets are contained in $s$, then the extension can be chosen to be a $\beta^{*}$-homeomorphism.

Proposition 3.3 (Wong [7]). For $X=Q$ or $s$, any homeomorphism $f$ of $X$ onto itself is isotopic to the identity mapping by means of an invertible-isotopy $\left\{f_{t}\right\}$ satisfying the following:

(1) if $X=Q$ and if $Q^{\prime} \subset Q$ is Core in $Q$ such that $f\left(Q^{\prime}\right) \subset s$, then we can require $f_{t}\left(Q^{\prime}\right) \subset s$ for all $t$ and

(2) if $X=Q$ and $f$ is a $\beta^{*}$-homeomorphism, then each $f_{t}$ is a $\beta^{*}$-homeomorphism. 
Note that properties (1) and (2) are not stated specifically in [7] but follow immediately from the construction.

\section{Proof of Theorem 1.2.}

Case 1. $X=Q$. By Proposition 3.2, there is an $f \in G(Q)$ such that $f(K) \subset s$. Let $g=f h f^{-1}$ and let $K_{1}=f(K) .\left.g\right|_{K_{1}}=$ identity. By Lemma 3.1 there is a sub-Hilbert cube $Q_{1} \subset s$ such that $K_{1} \subset Q_{1}$ and $g\left(Q_{1}\right) \subset s$. Then for some subset $Q^{\prime}$ of $Q$ which is Core in $Q, Q_{1} \subset Q_{1}^{\prime}$.

By Proposition 3.2 there is a $\beta^{*}$-homeomorphism $f_{1} \in G(Q)$ such that $f_{1}\left(Q_{1}\right)=Q_{1}^{\prime}$. Let $g_{1}=f_{1} g f_{1}^{-1}$. Since $f_{1}$ is $\beta^{*}, g_{1}\left(Q_{1}^{\prime}\right) \subset s$. By Proposition 3.3 there is an invertibleisotopy $\left\{g_{t}\right\}_{t \in I}$ between $g_{1}$ and $g_{0}=$ identity such that $g_{t}\left(Q_{1}^{\prime}\right) \subset s$ for all $t$. Let $K^{\prime}$ $=f_{1}\left(K_{1}\right), K^{\prime} \subset Q_{1}^{\prime} \subset s,\left.g_{1}\right|_{K^{\prime}}=$ identity. Let $G$ be the homeomorphism of $Q \times I$ onto $Q \times I$ defined by $\left.G\right|_{Q \times t}=g_{t}$. Then $G\left(K^{\prime} \times t\right) \subset s \times t$ for all $t$. Thus $G^{\prime}=\left.G\right|_{K^{\prime} \times I}$ satisfies the conditions that $G^{\prime}\left(K^{\prime} \times t\right) \subset s \times t$ and $\left.G^{\prime}\right|_{K^{\prime} \times\{0,1\}}=$ identity. By Theorem 2.1 , there is a homeomorphism $\psi$ of $Q \times I$ onto itself such that (1) $\psi(Q \times t)=Q \times t$ for all $t$, (2) $\left.\psi\right|_{K^{\prime} \times I}=G^{\prime}$ and (3) $\left.\psi\right|_{Q \times\{0,1\}}=$ identity. Denote $\psi$ by $\left\{\psi_{t}\right\}$ and denote $\psi_{t}^{-1} g_{t}$ by $\Phi_{t}$. Then (1) $\left.\Phi_{t}\right|_{K^{\prime}}=$ identity for all $t$ and (2) $\Phi_{1}=g_{1}, \Phi_{0}=$ identity. Let $h_{t}=$ $\left(f_{1} f\right)^{-1} \Phi_{t} f_{1} f .\left\{h_{t}\right\}$ is the desired isotopy.

Case 2. $X=s$. By Proposition 3.3 there is an invertible-isotopy $G=\left\{g_{t}\right\}$ of $s$ onto itself such that $g_{1}=h, g_{0}=$ identity. By Theorem 2.1 there is a homeomorphism $F$ of $s \times I$ onto itself satisfying (1), (2), and (3) of Theorem 2.1. Denote $\left.F\right|_{s \times t}$ by $f_{t}$ and denote $f_{t}^{-1} g_{t}$ by $h_{t}$. Then $\left\{h_{t}\right\}$ is the desired isotopy.

5. An example. The following example shows that the requirement of $K$ in Theorem 1.1 to have property-Z in $Q$ is sometimes necessary.

Write $Q=J_{1} \times J_{2} \times \cdots$ where each $J_{i}=[-1,1]=J$. Let $K$ be the set $\left\{\left(x_{1}, x_{2}, \ldots\right) \in Q \mid x_{1}=0\right\}$. $K$ does not satisfy property-Z in $Q$. Let $f \in G(Q)$ be the identity mapping and let $g \in G(Q)$ be defined by $g\left(x_{1}, x_{2}, \ldots\right)=\left(-x_{1}, x_{2}, x_{3}, \ldots\right)$. $f, g$ are $K$-coincident. But there exists no invertible-isotopy between $f$ and $g$ which is also $K$-stationary. To see this let us suppose the contrary. Let $F=\left\{f_{t}\right\}$ be such an isotopy. Let $P=(-1,0,0, \ldots) \in Q$ and let $\pi_{1}: Q \rightarrow J_{1}$ be the projection. Then $\pi_{1}(F(P \times I))=[-1,1]$. So for some $t, 0=\pi_{1} F(P \times t)=\pi_{1} f_{t}(P)$ contradict to the assumption that $\left.f_{t}\right|_{K}=$ identity. The following corollary clarifies the situation.

Corollary 5.1. Let $X=Q$ and let $f, g$ and $K$ be as above. Suppose $h \in G(X)$ such that $g, h$ are $K$-coincident, then either there is an invertible-isotopy between $f$ and $h$ which is $K$-stationary or there is an invertible-isotopy between $g$ and $h$ which is $K$ stationary.

Proof. Let $X_{1}=\left\{x \in Q \mid x_{1} \leqq 0\right\}, X_{2}=\left\{x \in Q \mid x_{1} \geqq 0\right\} . K=X_{1} \cap X_{2}$. Since $\left.h\right|_{K}$ $=$ identity, then either $h\left(X_{1}\right)=X_{1}$ or $h\left(X_{1}\right)=X_{2}$.

Case 1. $h\left(X_{1}\right)=X_{1}$. Let $h_{1}=\left.h\right|_{X_{1}}$ and $h_{2}=\left.h\right|_{X_{2}}$. For $i=1,2, K$ has property-Z in $X_{i}$. By Theorem 1.1 there is an invertible-isotopy $H_{i}=\left\{h_{t}^{i}\right\}$ of $X_{i}$ onto itself between $h_{i}$ and the identity such that $H_{i}$ is $K$-stationary. Define $H=\left\{h_{t}\right\}$ by $\left.h_{t}\right|_{x_{i}}=h_{t}^{i}$. 
Case 2. $h\left(X_{1}\right)=X_{2}$. Then $g h$ satisfies the assumption of Case 1. Let $H=\left\{h_{t}\right\}_{t \in I}$ be defined as in Case 1 for $g h$. Then $h_{t}^{\prime}=\left\{g h_{t}\right\}$ is the desired isotopy.

6. Throughout this section, let $N$ be the set of positive integers and we write $Q=\prod_{i \in N} J_{i}$. Let $\pi_{i}$ denote the projection of $Q$ onto $J_{i}$. If $\alpha_{1}, \alpha_{2} \subset N, \alpha_{1} \cup \alpha_{2}=N$, $\alpha_{1} \cap \alpha_{2}=\varnothing$ and both $\alpha_{1}, \alpha_{2}$ are infinite, then we say $Q$ factors into $Q_{1} \times Q_{2}$ where $Q_{1}=\prod_{i \in \alpha_{1}} J_{i}$ and $Q_{2}=\prod_{i \in \alpha_{2}} J_{i}$. Suppose $\alpha \subset N$ and $\left\{X_{i}\right\}_{i \geqq_{1}}$ are spaces. Let $h \in G\left(\prod_{i \in \alpha} X_{i}\right)$, then $h^{\prime}$ is a natural extension of $h$ onto $\prod_{i \in N} X_{i}$ if $h^{\prime}(x, y)=(h(x), y)$ for all $x \in \prod_{i \in \alpha} J_{i}$ and $y \in \prod_{i \notin \alpha} J_{i}$. We consider $Q$ as a convex subset of the linear space $\prod_{i=1}^{\infty} R_{i}, R_{i}=$ reals.

Proof of Theorem 2.1. The theorem is explicitly shown for $X=Q$. For $X=s$, note that when restricted to $s \times I$ the constructed homeomorphisms are the desired ones. A proof is given for the special case in which $Q$ factors into $Q_{1} \times Q_{2}$, and $K \cup h(K) \subset Q_{1} \times 0 \times I$. The proof is completed by reducing the general case to the special one.

Consider the following for the special case. Let $\varphi(t)=t(1-t), Q_{i}=J_{i 1} \times J_{i 2} \times \cdots$. Let $e: Q_{1} \rightarrow Q_{2}$ be the identification so that $\pi_{2 j} e(x)=\pi_{i j}(x)$. Let $H(J)$ be the space of homeomorphisms of the interval $J=[-1,1]$ leaving the endpoints fixed. $H(J) \cong s$ [4]. For $a, b \in \operatorname{Int}(J)$, let $p \omega(a, b, J)$ be the simple piecewise linear homeomorphism of $J$ taking $[-1, a]$ to $[-1, b]$ and $[a, 1]$ to $[b, 1]$. For $x \in Q$ and $t \in I, \varphi(t) \cdot x$ means coordinate multiplication.

Let $\bar{g}_{i}: Q_{1} \times I \rightarrow H\left(J_{2 i}\right)$ be the map where $\bar{g}_{i}(x, t)=p \omega\left(0, \varphi(t) \cdot \pi_{1 i}(x), J_{2 i}\right)$. Define $g$ a homeomorphism of $Q_{1} \times \prod_{i>0} J_{2 i} \times I$ by

$$
g\left(x_{1}, x_{21}, x_{22}, \ldots, t\right)=\left(x_{1}, \bar{g}_{1}\left(x_{1}, t\right)\left(x_{21}\right), \bar{g}_{2}\left(x_{1}, t\right)\left(x_{22}\right), \ldots, t\right) .
$$

Note that this homeomorphism is the identity for $t=0,1$, and the point $(x, 0, t)$ in $K$ is taken to $(x, \varphi(t) \cdot e(x), t)$.

Let $\tilde{k}_{i}: h(K) \cup Q_{1} \times 0 \times\{0,1\} \rightarrow H\left(J_{2 i}\right)$ be defined as

$$
\tilde{k}_{i}(x, 0, t)=p \omega\left(0, \varphi(t) \cdot \pi_{1 i} h^{-1}(x, 0, t), J_{2 i}\right) .
$$

Let $\tilde{k}$ having the same domain and going into $\prod_{i>0} H\left(J_{2 i}\right)$ be defined by the coordinate maps $\tilde{k}_{i}$. By Dugundji's theorem $\tilde{k}$ can be extended to all of $Q_{1} \times 0 \times I$. Call the extension $k$. Define $k$ a homeomorphism of $Q_{1} \times Q_{2} \times I$ by

$$
k\left(x_{1}, x_{2}, t\right)=\left(x_{1}, k\left(x_{1}, 0, t\right)\left(x_{2}\right), t\right) .
$$

Note that this homeomorphism is the identity for $t=0,1$, and the point $h(x, 0, t)$ in $h(K)$ is taken to $\left(\pi_{1} h(x, 0, t), \varphi(f) \cdot e(x), t\right)$.

Let $K^{\prime}$ be the projection of $g(K)$ into $0 \times Q_{2} \times I$, which is the same as the projection of $k h(K)$. For $t \neq 0,1$, these projections are one-to-one. Let $x=e^{-1}(y) / \varphi(t)$; the point $(0, y, t)$ in $K^{\prime}$ corresponds to $(x, y, t)$ in $g(K)$ and $\left(\pi_{1} h(x, 0, t), y, t\right)$ in $k h(K)$.

Let $f_{i}: K^{\prime} \cup 0 \times Q_{2} \times\{0,1\} \rightarrow H\left(J_{1 i}\right)$ be the map where

$$
\tilde{f}_{i}(0, y, t)=p \omega\left(\pi_{1 i} x, \pi_{1 i} h(x, 0, t), J_{1 i}\right),
$$


if $t \neq 0,1$ and $x=e^{-1}(y) / \varphi(t)$; and $\tilde{f}_{i}(0, y, t)=$ identity on $J_{1 i}$ if $t=0,1$. As in defining $\bar{k}$, define $\bar{f}: 0 \times Q_{2} \times I \rightarrow \prod_{i>0} H\left(J_{1 i}\right)$. Define $f$ a homeomorphism of $Q_{1} \times Q_{2} \times I$ by

$$
f\left(x_{1}, x_{2} t\right)=\left(\bar{f}\left(0, x_{2}, t\right)\left(x_{1}\right), x_{2}, t\right) .
$$

Note that this homeomorphism is the identity for $t=0,1$ and the point $(x, \varphi(t) \cdot e(x), t)$ in $g(K)$ goes to the point $\left(\pi_{1} h(x, 0, t), e(x) \cdot \varphi(t), t\right)$ in $k h(K)$.

The desired homeomorphism is $h_{1}=k^{-1} f g . h_{1}$ is level-preserving and the identity at $t=0,1 .\left.h_{1}\right|_{K}=h$ as follows. Let $(x, 0, t) \in K$ and $t \neq 0,1 . h_{1}(x, 0, t)=k^{-1} f g(x, 0, t)$ $=k^{-1} f(x, \varphi(f) \cdot e(x), t)=k^{-1}\left(\pi_{1} h(x, 0, t), \varphi(f) \cdot e(x), t\right)=h(x, 0, t)$.

In the general case with no condition on $K \cup h(K)$, there is a $\beta^{*}$-homeomorphism $l$ on $Q \times I$, where $Q=Q_{1} \times Q_{2}$, such that $l(K \cup h(K)) \subseteq Q_{1} \times 0 \times I$, and $l$ is independent of the $I$ coordinate [2]. $l h l^{-1}: l(K) \rightarrow \ln (K)$ satisfies the special case. If $h_{1}$ extends $l h l^{-1}$ then the desired extension is $l^{-1} h_{1} l$.

\section{REFERENCES}

0. R. D. Anderson, Hilbert space is homeomorphic to the countable infinite product of lines, Bull. Amer. Math. Soc. 72 (1966), 515-519. MR 32 \#8298.

1. - Topological properties of the Hilbert cube and the infinite product of open intervals, Trans. Amer. Math. Soc. 126 (1967), 200-216. MR 34 \#5045.

2. —, On topological infinite deficiency, Michigan J. Math. 14 (1967), 365-383. MR 35 \#4893.

3. - - Strongly negligible sets in Fréchet manifolds, Bull. Amer. Math. Soc. 75 (1969), 64-67. MR 38 \#6634.

4. - Spaces of homeomorphisms of finite graphs, Illinois J. Math. (to appear).

5. V. L. Klee, Convex bodies and periodic homeomorphisms in Hilbert space, Trans. Amer. Math. Soc. 74 (1953), 10-43. MR 14, 989.

6. K. Kuratowski, Topologie, vol. 2, 3rd ed., Monografie Mat., Tom 21, PWN, Warsaw, 1961, p. 32 (7). MR 24 \#A2958.

7. R. Y. T. Wong, On homeomorphisms of certain infinite dimensional spaces, Trans. Amer. Math. Soc. 128 (1967), 148-154. MR 35 \#4892.

8. — A A wild Cantor set in the Hilbert cube, Pacific J. Math. 24 (1968), 189-193. MR 36 \#4539.

UNIVERSITY OF CALIFORNIA,

Santa Barbara, California 93106 